\title{
DER EINFLUSS KLIMATISCHER FAKTOREN AUF DIE ENTWICKLUNG VON ERBSE
}

\author{
K. Multamäki \\ Zentrale für landwirtschaftliche Forschung, Abteilung für Pflanzenzüchtung, \\ Jokioinen
}

Eingegangen am 15, IX, 1961

In Finnland ist die Bedeutung der klimatischen Faktoren für die Entwicklung der wichtigsten Kulturpflanzen von Meteorologen und Landbauforschern - je nach der Pflanzenart mehr oder minder eingehend - beleuchtet worden $(7,8,9$, $13,14,16,19,20,21,22,23,24,25)$. Entsprechende Untersuchungen hat man im Auslande schon längst ausgeführt (vgl. 11, 19, 25). In derartigen Versuchen hat man u.a. zeigen können, dass zwischen den Wetterkomponenten und dem Wachstum der Erbse deutliche Abhängigkeitsverhältnisse festzustellen sind $(2,3,4,11$, $13,14,16,17)$.

In vorliegender Untersuchung kommt es in erster Linie darauf an, den Einfluss der klimatischen Faktoren auf den Entwicklungsrhythmus der Erbse unter finnischen Anbauverhältnissen zu erläutern. Die Untersuchung gründet sich auf Ergebnisse, die in den Erbsenversuchen der Abteilung für Pflanzenzüchtung in den Jahren $1929-60$ in Jokioinen $\left(60^{\circ} 49^{\prime}\right.$ n.Br., $23^{\circ} 30^{\prime}$ ö.L.) erhalten worden sind. In diesen Versuchen hat die Standardsorte Torsdags II aus Svalöf schon lange eine zentrale Stellung eingenommen; diese Sorte hat ja schon seit 1929 in Jokioinen ununterbrochener Erforschung unterstanden.

Die betreffenden Erbsenversuche sind beinahe die ganze Zeit — von 1929 bis 1959 - unter der Leitung desselben Züchters, nämlich des ehemaligen, langjährigen Direktors der Abteilung, Prof. Dr. V. A. Pesola, ausgeführt worden. Während dieser 31 Jahre hat Prof. Pesola alle wichtigsten Feldbeobachtungen der Erbsenversuche selbst vorgenommen. Weiter hat er die Untersuchungen über die Qualität (Kochbarkeit und Geschmack) geplant und persönlich überwacht. Von den übrigen Züchtern der Anstalt hat besonders Diplomlandwirt O. InKILÄ, der seit 1946 als Assistent Prof. Pesolas tätig gewesen ist, an der Arbeit zur Züchtung und Untersuchung von Erbse teilgenommen. 


\section{Versuchsverhältnisse}

Die Bodenart der Versuchsfelder ist humoser schwerer Ton, dessen Azidität zwischen $\mathrm{pH} 5.1$ und 6.1 variiert. Als Düngung erhielt das Versuchsfeld 150-600 $\mathrm{kg} / \mathrm{ha}$ Superphosphat, $80-200 \mathrm{~kg} / \mathrm{ha}$ Kalisalz $(40 \%$ oder $50 \%)$ und in einigen Jahren als Kopfdüngung 75-100 kg/ha Kalksalpeter. Die Grösse der Teilstücke betrug meistens $15 \mathrm{~m}^{2}$ und die Anzahl der Wiederholungen 5 oder 6 .

Ưber die Witterungsverhältnisse in den Jahren 1929-60 in Jokioinen und über ihren Einfluss auf das Wachstum der Erbse mag übersichtsweise folgendes angeführt werden. Unter den 32 Vegetationsperioden waren 23 solche, die in bezug auf Temperatur und Niederschlagsverhältnisse als ziemlich oder in einigen Jahren sogar sehr günstig für den Erbsenbau angesehen werden können. Dagegen kamen in sieben Sommern $(1936,1939$ - 41, 1946, 1947 und 1951) schwere Trockenperioden vor; durch sie erlitt das ganze Wachstum der Erbse beträchtliche Schädigungen. Anderseits mag die Entwicklung der Erbse auch durch ziemlich kühle Regenperioden erschwert worden sein. So verspätete sich aus diesem Grunde das Reifen der Bestände in hohem Grade in den Jahren 1943 und 1958.

In einigen Jahren traten während der Wachstumsperiode Nachtfröste auf. Es stellte sich dabei heraus, dass die Erbse in jungen Entwicklungsstufen verhältnismässig frosthart ist. In bezug auf Frosthärte sind zwischen den einzelnen Erbsensorten deutliche Unterschiede festzustellen gewesen (16).

Der gestreifte Blattrandkäfer (Sitona lineatus) ist oft auf jungen Saatpflanzen der Erbse als Schädling vorgekommen, aber die von ihm verursachten Beeinträchtigungen sind im allgemeinen gering geblieben. In Hülsen der Erbse sind jedes Jahr Beschädigungen, veranlasst durch die Larve des Erbsenwicklers (Laspeyresia nigricana), aufgetreten. Die Grösse dieser Schäden hat in den verschiedenen Jahren gewechselt, doch sind sie meistens bedeutungslos für die Brauchbarkeit der Ertragsresultate gewesen $(5,6,13)$. Die durch den Erbsenwickler verursachten Nachteile waren in den Jahren 1946 und 1959 ausnahmsweise stark. Die in vielen Jahren beobachtete, meistens aber verhältnismässig geringe Schädigung durch den Erbsenblasenfuss (Kakothrips robustus) hat vorzugsweise die späten Sorten betroffen (6).

\section{Material und Methoden}

Für das Erforschen der Entwicklung der Erbse und ihrer Abhängigkeit von Witterungsverhältnissen bot die Erbse Torsdags II ein reichliches Ergebnismaterial. Bei der Betrachtung des Entwicklungsrhythmus dieser Zuchtsorte wurde ihre Wachstumsdauer (W), in Tagen ausgedrückt, in zwei Phasen eingeteilt. Die erste Phase (I) umfasste die Aufblühzeit, das ist die Zeitspanne von der Aussaat bis zum Beginn des Blühens (einschliesslich des Aussaat- und des Blühbeginntages). Als Blühbeginn wurde - auf einem $15 \mathrm{~m}^{2}$ grossen Teilstück - das Auftreten der 810 ersten blühenden Erbsenindividuen festgelegt (12), als zweite Phase (II) der Rest der Wachstumsdauer bis zum Reifetage. Als reif wurde ein Erbsenindividuum dann beurteilt, nachdem seine untersten Hülsen an der Oberfläche lederartig und gelb geworden waren (12). 
In bezug auf die genannten drei Perioden wurden die jährlichen und durchschnittlichen Werte sowohl für die Dauer in Tagen als für die Mitteltemperaturen, die effektiven Temperatursummen und die Niederschlagssummen berechnet (Tabelle 1). Dabei ist die effektive Temperatursumme einer jeden Periode durch Addieren der täglichen effektiven Mitteltemperaturen erhalten worden. Bei ihrer Berechnung subtrahierte man $4^{\circ} \mathrm{C}$ von der auf übliche Weise berechneten Mitteltemperatur des Tages (vgl. 4, 23). Die Wetterbeobachtungen wurden unweit der Versuchsfelder gemacht. Die Temperatur wurde dabei in einer Hütte 2 Meter über dem Erdboden gemessen.

Bei Untersuchung der Nutzungseigenschaften der Erbse wurden sowohl die Kochbarkeit als der Geschmack der Samen bestimmt. Zur Erforschung der ersteren Eigenschaft lagen die zu untersuchenden Samen zuerst über Nacht in kaltem Wasser. Am folgenden Morgen kochte man die Erbsen so lange, bis sie weich geworden waren; die Kochdauer in Minuten wurde dann vermerkt $(13,18)$.

Für die Geschmacksuntersuchungen wurden die weichgekochten Erbsen der obigen Kochversuchte benutzt. Zur Anwendung kam eine Geschmacksskala von $1-10$ in der Weise, dass mit 10 eine solche Erbse bezeichnet wurde, die einen feinen, süssen Geschmack hatte, mit 4 dagegen eine Erbse, die deutlich bitter schmeckte.

Bei Auswertung des zur Verfügung stehenden Zahlenmaterials wurde von statistischen Methoden nach BonNiER und Tedin Gebrauch gemacht (1).

\section{Ergebnisse der Untersuchungen}

Aus Tabelle 1 ist zunächst ersichtlich, dass die Erbse Torsdags II in Jokioinen in den Jahren 1929-60 durchschnittlich am 14. Mai ausgesät worden ist. Die durch die Witterungsverhältnisse bedingte Aussaatszeit hat während der betreffenden Jahresfolge beträchtlich gewechselt, vom 25. April (1953) bis zum 27. Mai (1944 und 1955). Die Variationsbreite dieses Zeitpunktes hat sich somit auf 33 Tage belaufen.

Entsprechend kamen in den einzelnen Jahren bedeutende Unterschiede auch in anderen Entwicklungsvorgängen der Erbse vor. So variierte der Zeitpunkt des Blühbeginns um 24 Tage, vom 21. Juni im J. 1953 bis zum 14. Juli im. J. 1955. Noch mehr, 38 Tage, betrug die Variation im Reifedatum. Am frühesten oder am 25. Juli gelangte die Erbse Torsdags II im J. 1937 zur Reife, am spätesten dagegen im J. 1958, wo sie am 31. August als vollreif vermerkt wurde.

Aus Tabelle 1 ist zu ersehen, dass die Schwankungen in den Mitteltemperaturen von Jahr zu Jahr relativ gering gewesen sind. Dagegen zeigten die Niederschlagsmengen der verschiedenen Vegetationsperioden eine bedeutende Variabilität. Verhältnismässig wenig veränderlich waren wiederum die effektiven Temperatursummen, von denen die der ersten Entwicklungsphase durchschnittlich $434.6^{\circ} \mathrm{C}$ betrug, während die der zweiten Phase $452.1^{\circ} \mathrm{C}$ ausmachte. Der Variationskoeffizient des letzteren Mittelwertes erwies sich als beinahe zweimal so gross wie der von Phase I. Der Variationskoeffizient der effektiven Temperatursumme während der ganzen Wachstumsdauer (durchschn. $886.7^{\circ} \mathrm{C}$ ) näherte sich dem der ersten Phase. 
Tabelle 1. Die klimatischen Faktoren und die Dauer in Tagen während der ganzen Wachstumsdauer (W) und ihrer zwei Phasen (I, II) sowie die Aussaatzeiten und Samenerträge der Erbse Torsdags II in Jokioinen in den Jahren $1929-1960$

\begin{tabular}{|c|c|c|c|c|c|c|c|c|c|c|c|c|c|c|}
\hline \multirow[t]{2}{*}{ Jahr } & \multirow[t]{2}{*}{$\begin{array}{c}\text { Aussaat- } \\
\text { zeit }\end{array}$} & \multicolumn{3}{|c|}{$\begin{array}{c}\text { Mitteltemperatur } \\
\left({ }^{\circ} \mathrm{C}\right)\end{array}$} & \multicolumn{3}{|c|}{$\begin{array}{l}\text { Niederschlags- } \\
\text { summe (mm) }\end{array}$} & \multicolumn{3}{|c|}{$\begin{array}{l}\text { Effektive Tempe- } \\
\text { ratursumme }\left({ }^{\circ} \mathrm{C}\right)\end{array}$} & \multicolumn{3}{|c|}{$\begin{array}{l}\text { Dauer in } \\
\text { Tagen }\end{array}$} & \multirow{2}{*}{$\begin{array}{c}\text { Samen- } \\
\text { ertrag } \\
(\mathrm{kg} / \mathrm{ha}\end{array}$} \\
\hline & & I & II & W & I & II & W & I & II & W & I & II & W & \\
\hline 1929 & 17.5 & +12.3 & +14.4 & +13.3 & 155.7 & 123.3 & 279.0 & 454.2 & 508.2 & 962.4 & 55 & 49 & 104 & 3036 \\
\hline 1930 & 7.5 & +12.9 & +17.0 & +14.7 & 62.6 & 89.9 & 152.5 & 435.3 & 492.9 & 928.2 & 49 & 38 & 87 & 2647 \\
\hline 1931 & 9.5 & +11.3 & +16.2 & +13.3 & 63.2 & 109.5 & 172.7 & 431.4 & 464.5 & 895.9 & 59 & 37 & 96 & 3253 \\
\hline 1932 & 19.5 & +12.2 & +17.6 & +14.4 & 68.6 & 75.8 & 144.4 & 432.3 & 461.0 & 893.3 & 50 & 33 & 83 & 1514 \\
\hline 1933 & 16.5 & +12.4 & +16.0 & +14.1 & 22.7 & 129.4 & 152.1 & 416.4 & 469.2 & 885.6 & 49 & 39 & 88 & 2091 \\
\hline 1934 & 7.5 & +11.3 & +17.2 & +13.6 & 76.8 & 106.2 & 183.0 & 413.9 & 487.9 & 901.8 & 57 & 37 & 94 & 2428 \\
\hline 1935 & 15.5 & +12.0 & +15.2 & +13.3 & 61.2 & 46.4 & 107.6 & 399.6 & 403.2 & 802.8 & 50 & 36 & 86 & 1711 \\
\hline 1936 & 15.5 & +14.2 & +17.2 & +15.5 & 83.9 & 6.7 & 130.6 & 439.2 & 423.8 & 863.0 & 43 & 32 & 75 & 693 \\
\hline 1937 & 5.5 & +13.2 & +17.1 & +14.7 & 90.8 & 2.7 & 143.5 & 471.5 & 404.9 & 876.4 & 51 & 31 & 82 & 1623 \\
\hline 1938 & 7.5 & +11.3 & +1 & $+1:$ & 96.2 & 46.6 & 142.8 & 438.0 & 439.0 & 877.0 & 60 & 30 & 90 & 2290 \\
\hline 1939 & 16.5 & +12.0 & +16.6 & +15.1 & 30.1 & 139.6 & 169.7 & 369.8 & 502.1 & 871.9 & 46 & 40 & 86 & 620 \\
\hline 1940 & 18.5 & +12.7 & +16.3 & +13.8 & 39.5 & 90.3 & 129.8 & 427.6 & 392.4 & 820.0 & 49 & 32 & 81 & 961 \\
\hline 1941 & 22.5 & +12.8 & +18.8 & +14.9 & 42.1 & 3.0 & 45.1 & 405.6 & 370.6 & | 776.2 & 46 & 25 & 71 & 937 \\
\hline 1942 & 15.5 & +12.4 & +14.7 & +13.4 & 77.4 & 140.8 & 218.2 & 467.8 & 490.0 & & 56 & 46 & 102 & $\overline{1676}$ \\
\hline 1943 & 8.5 & +12.0 & +15.3 & +13.4 & 114.0 & 258.4 & 372.4 & 422.0 & 531.1 & & 54 & 47 & 101 & 1100 \\
\hline 1944 & & +13.1 & +16.0 & +14.5 & 86.0 & 110.0 & 196.0 & 430.0 & 490.8 & & 47 & 41 & 88 & 1590 \\
\hline 1945 & & +11.9 & +18.2 & +14.0 & 88.0 & & 160.2 & 450.1 & 410.8 & 860.9 & 57 & 29 & 86 & 1287 \\
\hline 1946 & & & +17.2 & +15.4 & 41.8 & & & 469.0 & & & 46 & 31 & 77 & 1388 \\
\hline 1947 & 17.5 & +13.2 & +15.6 & +14.3 & 45.4 & & 107.0 & 405.4 & 404.4 & & 44 & 35 & 79 & 1243 \\
\hline 1948 & 12.5 & +13.0 & +15.3 & +13.9 & 113.2 & & 202.3 & 478.9 & 405.9 & & 53 & 36 & 89 & 3344 \\
\hline 1949 & 16.5 & +13.8 & +14.7 & +14.2 & 71.9 & & & 490.7 & 451.3 & & 50 & 42 & 92 & 3017 \\
\hline 1950 & 15.5 & +11.8 & +15.0 & +13.1 & 66.4 & 41.0 & 107.4 & 413.1 & 407.5 & & 53 & 37 & 90 & 1506 \\
\hline 1951 & 19.5 & +11.2 & +14.8 & +12.5 & 56.9 & 54.8 & 111.7 & 402.1 & 357.6 & 759.7 & 56 & 33 & 89 & 1232 \\
\hline 1952 & 9.5 & +10.5 & +14.7 & +12.2 & 84.4 & 115.7 & 200.1 & 391.4 & 448.4 & 839.8 & 60 & 42 & 102 & 2481 \\
\hline 1953 & 25.4 & +11.2 & +16.6 & +13.3 & 95.7 & 136.6 & 232.3 & 415.5 & 478.9 & 894.4 & 58 & 38 & 96 & 1983 \\
\hline 1954 & 12.5 & +12.7 & +15.6 & +14.1 & 35.1 & 201.9 & 237.0 & 471.1 & 533.9 & 1005.0 & 54 & 46 & 100 & 1504 \\
\hline 1955 & 27.5 & +12.2 & +16.6 & +13.9 & 45.8 & 7.0 & 52.8 & 403.3 & 366.1 & 769.4 & 49 & 29 & 78 & 1953 \\
\hline 1956 & 15.5 & +13.0 & +13.8 & +13.4 & 68.4 & 117.3 & 185.7 & 449.7 & 461.4 & 911.1 & 50 & 47 & 97 & 1650 \\
\hline 1957 & 16.5 & +11.6 & +16.9 & +13.7 & 84.1 & 140.4 & 224.5 & 449.1 & 489.8 & 938.9 & 59 & 38 & 97 & 2540 \\
\hline 1958 & 26.5 & +13.6 & +13.8 & +13.8 & 57.1 & 110.5 & 167.6 & 469.4 & 487.5 & 956.9 & 49 & 49 & 98 & 1336 \\
\hline 1959 & 2.5 & +11.1 & +16.8 & +13.1 & 59.6 & 25.0 & 84.6 & 428.7 & 421.7 & 850.4 & 60 & 33 & 93 & 2266 \\
\hline 1960 & 13.5 & +13.9 & +16.6 & +15.2 & 59.8 & 142.0 & 201.8 & 465.0 & 602.4 & 11067.4 & 47 & 48 & 95 & 2211 \\
\hline
\end{tabular}

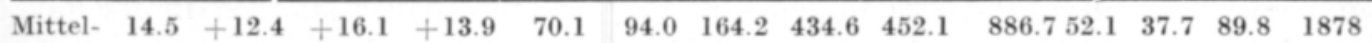
wert

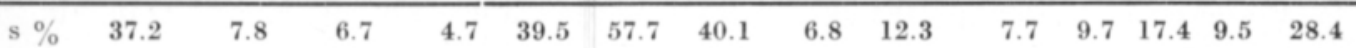

In entsprechender Weise schwankte die Dauer der zweiten Phase (durchschn. 37.7 Tage) deutlich mehr als die von Phase I (52.1 Tage) und die der ganzen Wachstumsdauer (89.8 Tage). Die Variationsbreite der letzgenannten Periode belief sich auf 34 Tage: von 71 Tagen im J. 1941 bis zu 104 Tagen im J. 1929. 
Vergleichsweise wurden auch die auf den Gefrierpunkt bezogenen Temperatursummen für die drei Entwicklungsperioden berechnet. Die betreffende Temperatursumme von Phase I belief sich durchschnittlich auf $642.8^{\circ} \mathrm{C}$ mit $\mathrm{s}=5.7 \%$; die der zweiten Phase auf $602.9^{\circ} \mathrm{C}(12.9 \%)$ und die der ganzen Wachstumsdauer auf $1245.7^{\circ}(7.6 \%)$. Die Mindest- und Höchstwerte der Temperatursumme der ganzen Wachstumsdauer betrugen $1060.2^{\circ} \mathrm{C}$ im J. 1941 und $1447.4^{\circ} \mathrm{C}$ im J. 1960.

Ein Vergleich zwischen den Witterungsverhältnissen u.a. der zwei Entwicklungsphasen I und II zeigt, dass Phase II durchschnittlich um $3.7^{\circ} \mathrm{C}\left({ }^{* * *}\right)$ wärmer gewesen ist. Ausserdem war ihre mittlere Niederschlagsmenge um $23.9 \mathrm{~mm}\left({ }^{*}\right)$ höher. Die erste Phase dauerte im Durchschnitt 14.4 Tage $(* * *)$ länger als die zweite. Der Anteil der zweiten Phase an der ganzen Wachstumsdauer, in Tagen ausgedrückt, betrug $42 \%$.

Zwischen den effektiven Temperatursummen der beiden Phasen war keine gesicherte Differenz festzustellen. Dagegen überschritt die von der ersten Phase beanspruchte, auf den Gefrierpunkt bezogene Temperatursumme bedeutsam die von Phase II um $39.9^{\circ} \mathrm{C}\left({ }^{*}\right)$. Der Anteil der zweiten Phase an der ganzen Wachstumsdauer, auf diese Weise berechnet, belief sich auf $48 \%$.

Der Samenertrag der Erbse Torsdags II überschritt in vier Jahren $3000 \mathrm{~kg} / \mathrm{ha}$. In drei Vegetationsperioden blieben die Erntemengen geringer als $1000 \mathrm{~kg} / \mathrm{ha}$. Die Mindest- und Höchsterträge dieser Sorte beliefen sich auf $693 \mathrm{~kg} / \mathrm{ha}$ im J. 1936 und $3344 \mathrm{~kg} / \mathrm{ha}$ im J. 1948. Der durchschnittliche Ertrag machte $1878 \mathrm{~kg} / \mathrm{ha}$ aus.

Zur Ermittlung etwaiger Wechselbeziehungen in dem zur Verfügung stehenden Zahlenmaterial wurden insgesamt 37 Korrelationskoeffizienten berechnet (Tabelle 2). Meistens wurde dabei die Abhängigkeit des Entwicklungsrhythmus und des Samenertrages von den Witterungsverhältnissen untersucht.

Aus Tabelle 2 ist zunächst ersichtlich, dass der Zeitpunkt der Aussaat in einer negativen Korrelation zur Dauer der ersten Phase steht. Dieses Verhältnis bedeutet, dass je später die Aussaat ausgeführt wird, die erste Phase um so kürzer ausfällt. Der Wechsel des Aussaattages übte dagegen weder auf die Länge der zweiten Phase noch auf die der ganzen Wachstumsdauer einen gesicherten Einfluss aus.

Zwischen den verschiedenen klimatischen Faktoren und der Dauer der drei untersuchten Entwicklungsperioden waren mehrere bedeutsame Korrelationen zu erkennen. Am deutlichsten schien eine derartige Dauer von der Mitteltemperatur abhängig zu sein: in allen drei Perioden äusserte sich in dieser Hinsicht eine negative Wechselbeziehung. Die effektiven Temperatursummen sowie die Niederschlagssummen der zweiten Phase und der ganzen Wachstumsdauer standen ihrerseits in positiver Korrelation zu der Dauer der betreffenden Perioden.

Besonders für den praktischen Anbau interessant sind die Korrelation, die sich auf den Samenertrag beziehen. Es wäre ja, z.B. vom Standpunkte des Landwirtes aus betrachtet, wünschenswert, die Ertragsmenge schon während der Vegetationsperiode auf Grund der klimatischen Faktoren u.dgl. möglichst genau vorhersagen zu können. Unter den 14 berechneten Wechselbeziehungen dieser Art gab es jedoch nur drei bedeutsame. Es stellte sich heraus, dass, je länger Phase I und die ganze Wachstumsdauer währten, die Sameneträge um so grösser ausfielen. Zwischen 

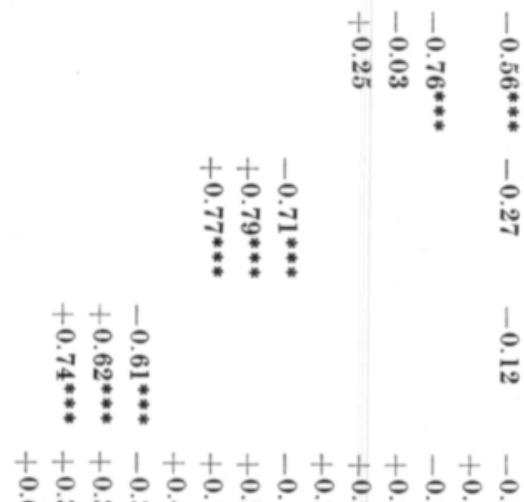

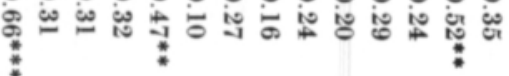

Dauer der ersten Phase (I),

Tage

Dauer der zweiten Phase (II),

Tage

$$
\text { !o }
$$

중 
den Stroh- und Samenerträgen trat auch eine positive Wechselbeziehung hervor. Dagegen liess sich zwischen dem Aussaattag oder den klimatischen Faktoren einerseits und dem Samenertrag anderseits keine gesicherte Korrelation ermitteln.

Viel leichter scheint es zu sein, die Grösse des Strohertrages im voraus zu schätzen. Unter den vier diesbezüglichen Korrelationen waren nicht weniger als drei bedeutsam. Sowohl die Länge der Wachstumsdauer als auch ihre effektive Temperatursumme und ihre Niederschlagssumme schienen in positiver Wechselbeziehung zu dem Strohertrage zu stehen. Dagegen war die Mitteltemperatur während der Wachstumsdauer in dieser Hinsicht wirkungslos.

Wie zu erwarten, war zu erkennen, dass die Pflanzenhöhe und die Standfestigkeit in negativer Korrelation zueinander stehen.

Bei Untersuchung der Korrelationsverhältnisse hinsichtlich einiger Nutzungseigenschaften der Erbse Torsdags II stellte es sich heraus, dass Schwankungen im 1000-Korngewicht keine gesicherte Wirkung auf Kochdauer und Geschmack ausübten. Der Geschmack seinerseits war unabhängig von Schwankungen in der Kochdauer.

Einige Koeffizienten in Tabelle 2 erhellen den Zusammenhang zwischen den untersuchten klimatischen Faktoren. Es stellt sich heraus, dass die Niederschlagssumme der Wachstumsdauer parallel mit der effektiven Temperatursumme derselben Periode variiert. Zwischen der Niederschlagssumme und der Mitteltemperatur der Wachstumsdauer sowie zwischen der Mitteltemperatur und der effektiven Temperatursumme desselben Zeitabschnittes sind dagegen keine bedeutsamen Wechselbeziehungen festzustellen gewesen.

\section{Besprechung der Ergebnisse}

Die oben angeführten Ergebnisse beziehen sich auf eine Sorte, die Svalöfer Erbse Torsdags II. Diese Züchung ist in den 1930er und 1940er Jahren in relativ grossem Masse in Finnland angebaut worden und ist hier jahrelang - zusammen mit der Erbse Torsdags I - die häufigste Erbsensorte gewesen (14, 15, 26). Seit etwas mehr als zehn Jahren begann sich ihre Anbaufläche infolge des Erscheinens neuerer, ertragreicherer Sorten bedeutend zu vermindern, und zurzeit ist sie ganz gering (10). Die mittelfrühe Torsdags II ist als eine anspruchslose, verhältnismässig dürreresistente und ziemlich ergiebige Erbse anzusehen (15).

In vorliegender Untersuchung werden Eigenschaften und Wechselbeziehungen einer einzigen Zuchtsorte, der Torsdags II, betrachtet. Ferner ist für die vorliegende Studie nur von einer Versuchsstelle herrührendes Forschungsmaterial benutzt worden. Die in Jokioinen bei Torsdags II gewonnene langjährige Erfahrung umfasst jedoch ein so reichliches Ergebnismaterial, dass sich wohl auf seiner Grundlage zuverlässige Schlüsse über den Entwicklungsrhythmus sowie verschiedene Korrelationen bei den mittelfrühen Erbsensorten in weitem Gebiet Südwestfinnlands ziehen lassen. Ausserdem können die erlangten Untersuchungsergebnisse sicherlich einige der wichtigsten Züge der Reaktionsnorm der Erbse in finnischen Anbauverhältnissen im allgemeinen erhellen. 
Gegen die in vorliegender Untersuchung verwendeten Temperaturwerte kann bemerkt werden, dass sie sich nicht auf Messungen im Bestande gründen. Es liegt ja auf der Hand, dass Differenzen zwischen den Hüttenwerten und dem Mikroklima den Bestandes vorliegen. Die angeführten Temperatursummen sind daher als einigermassen relativ zu betrachten. Trotz dieses Mangels vermögen die gegebenen Werte offenbar die charakteristische Wirkung der Temperatur auf die Entwicklung der Erbse zu beleuchten (vgl. 4, 11).

Bei Forschungen über die Variabilität der Temperatursumme und der in Tagen angegebenen Länge der Wachstumsdauer bei Sommergetreidearten in Finnland hat man feststellen können, dass die erstere Menge bedeutend weniger wechselt $(19,23,25)$. In der vorliegenden Untersuchung über Erbse hat sich ein ähnliches Resultat herausgestellt. Die Variationskoeffizienten der auf beiderlei Weise berechneten Temperatursummen erwiesen sich als beinahe gleich gross. Die Temperatursummen variierten deutlich weniger als die Längen der drei Entwicklungsperioden. Deshalb kann man wohl auch in bezug auf die Erbse in finnischen Anbauverhältnissen als festgestellt ansehen, dass die für eine Entwicklungsphase erforderliche Temperatursumme beständiger als die entsprechende Anzahl der Wachstumstage ist. $\mathrm{Zu}$ gleichsinnigen Ergebnissen über die Temperatursummen einiger Kulturpflanzen ist man schon früher im Auslande gekommen, wo diese Frage besonders wegen ihrer grossen Bedeutung für die Konservenindustrie eingehend erforscht worden ist. Zurzeit werden auch die dabei gewonnenen Erfahrungen in der genannten Industrie in grossem Umfang in die Praxis umgesetzt (11).

Die durchschnittliche auf den Gefrierpunkt bezogene Temperatursumme der ganzen Wachstumsdauer $\left(1245.7^{\circ}\right)$ der Sorte Torsdags II ist als sehr niedrig zu betrachten. Nach einigen Versuchsergebnissen aus Jokioinen halten sich nur die allerfrühesten Getreidesorten in dieser Hinsicht auf demselben Niveau. Als solche wurden eine Landsorte des Sommerweizens Kerimäkeläinen (25) und die Gerstenzüchtungen Olli und Vega (19) festgestellt. Die Anbausicherheit der Erbse Torsdags II hat sich auch in Mittelfinnland als genügend bewährt (14).

Bei der Betrachtung der berechneten Korrelationen (Tabelle 2) fällt zuerst auf, dass die Variation der klimatischen Faktoren verhältnismässig oft eine gesicherte Einwirkung auf die Dauer der drei Entwicklungsperioden ausübt. So kann man z.B. vorhersagen, dass, je höher sich die Mitteltemperatur während einer Entwicklungsperiode bei der Erbse Torsdags II hält, die betreffende Periode eine um so kürzere Zeit währen wird. In einigen Untersuchungen anderer Forscher hat man bei manchen Erbsensorten analoge Resultate über die Wirkung der Temperatur erhalten $(3,4,11,13)$. Eine ähnliche Wechselbeziehung ist des weiteren bei Sommergetreidearten in finnischen Versuchen festgestellt worden $(19,22,23,24$, 25). Anderseits hat man hier u.a. eine verzögernde Einwirkung des Niederschlags auf die Entwicklung der Erbse $(13,16)$ sowie der Sommergetreidearten $(19,22,23)$ ermittelt.

Zwischen den Wetterkomponenten und dem Samenertrag der Erbse Torsdags II konnte kein deutliches Abhängigkeitsverhältnis herausgestellt werden. Dieses Resultat bedeutet, dass es sich nicht als möglich erwiesen hat, auf Grund klimatischer Faktoren der Wachstumsperiode die Menge des Samenertrages direkt 
vorauszuschätzen. Dagegen kann man für diesen Zweck die Dauer der ersten Phase und die der ganzen Wachstumsdauer mit offenbarer Sicherheit anwenden.

Im Gegensatz zum Samenertrage tritt die Abhängigkeit des Strohertrages von klimatischen Faktoren in deutlichen Korrelationen hervor. Ferner äusserte sich zwischen den Samen- und den Stroherträgen eine positive Wechelbeziehung. Dieses Verhältnis zeigt, dass - zum mindesten bei der Erbse Torsdags II — der Strohertrag parallel mit dem Samenertrage variiert, ohne sich offenbar auf dem Kosten des Samenertrages in kühlen und regnerischen Sommern ausserordentlich üppig zu entwickeln.

Zwischen der Niederschlagssumme und der effektiven Temperatursumme der ganzen Wachstumsdauer ist eine positive Korrelation festgestellt worden. Eine entsprechende Abhängigkeit hat man auch in einigen finnischen Versuchen mit Sommergetreidearten mehr oder minder deutlich beobachtet $(19,23,24)$.

\section{Schlussfolgerungen}

Im Entwicklungsrhythmus der Erbse Torsdags II ist in Jokioinen während der 32 untersuchten Wachstumsperioden eine bedeutende variablilität hervorgetreten.

Unter den klimatischen Faktoren haben die Mitteltemperaturen relativ wenig, die Niederschlagsmengen dagegen in hohem Grade geschwankt. Die Variabilität in den Temperatursummen der Entwicklungsperioden erwies sich als geringer als die in der Anzahl der Tage. Die Schwankungen waren am grössten in den Temperatursummen und in der Anzahl der Wachstumstage der zweiten Entwicklungsphase.

Die Abhängigkeit des Entwicklungsrhythmus von den Wetterkomponenten äusserte sich deutlich in gesicherten Korrelationen zwischen den klimatischen Faktoren und der Dauer der drei Entwicklungsperioden.

Zwischen der Niederschlagssumme und der effektiven Temperatursumme der Wachstumsdauer zeigte sich eine positive Wechselbeziehung.

Zwischen der Dauer der ersten Phase und der ganzen Wachstumsdauer einerseits und dem Samenertrage anderseits wurden positive Korrelationen festgestellt. Der Strohertrag stand in positiver Korrelation sowohl zu der Länge, der Niederschlagssumme und der effektiven Temperatursumme der Wachstumsdauer als auch zum Samenertrag.

Höhe und Standfestigkeit der Bestände zeigten eine negative Korrelation. Zwischen Grösse und Nutzungseigenschaften des Samens konnten keine bedeutsamen Korrelationen festgestellt werden.

\section{LITERATUR}

(1) Bonnier, G. \& Tedin, O. 1957. Biologisk variationsanalys. $186 \mathrm{~s}$. Stockholm.

(2) Fuchs, W. H. 1941. Beobachtungen an einem Erbsenaussaatzeitenversuch. Angew. Bot. 23: $342-347$.

(3) - - 1943. Aussaatzeit und Entwicklungsgeschwindigkeit bei Gemüseerbsen. Pfl.bau 19: 216-220. 
(4) Fuchs, W. H. \& Müнlendycк, E. 1951. Uber den Einfluss der Aussaatzeit und der Temperatur auf die Entwicklung von Erbsensorten. Z. Pfl.zücht. 30: 172-187.

(5) INкıL.̈, O. 1948. Hernekääriäisen (Grapholitha nigricana Steph.) peltoherneelle aiheuttamista vahingoista. Summary: Damages caused by Grapholitha (Grapholitha nigricana Steph.) to field peas. Maatal.tiet. aikak. 9: 58-66.

(6) - 1960. Peltoviljelyn tuhohyönteisistä Jokioisissa. Referat: Om skadeinsekter på åker och ång i Jockis. Lounais-Hämeen luonto 8: 29-33.

(7) KERÄNEN, J. 1931. Kasvukauden săiden ja vuodentulon keskinäisestä riippuvaisuudesta maassamme vuosina 1921-28. Taloudellisen Neuvottelukunnan julk. 13: 1-38.

(8) - - 1931. Vuodentulon riippuvaisuudesta kasvukauden lämpö- ja sadeoloista Suomen eri lääneissä. I. Korrelatiotekijät. Referat: Uber die Abhängigkeit der Ernteerträge von den Temperaturen und Regenmengen während der Vegetationszeit in Finnland. Acta agr. fenn. 23: 1-31.

(9) - 1931. Uber die Temperaturverhältnisse in Finnland während der Vegetationszeit an den Nordgrenzen der wichtigsten Kulturpflanzen. Gerlands Beitr. Geophysik 33: 261-267.

(10) Maataloushallitus, Tilastotoimisto. 1961. Maataloustilastollinen kuukausikatsaus 4: 68-76.

(11) Otroson, L. 1958. Growth and maturity of peas for canning and freezing. Växtodling 9: 1-112.

(12) PesolA, V. A. 1924. Kenttäkoeopas. 116 s. Porvoo.

(13) - - 1935. Peltoherneen jalostuksesta ja sen tuloksista. Referat: Ủber die Erbsenzüchtung der landwirtschaftlichen Versuchsanstalt Finnlands, Abt. für Pflanzenzüchtung, und ihre Ergebnisse. Valt. maatal.koetoim. julk. 66: 1-91.

(14) - - 1941. Suomen kasvinviljelysalueet. Referat: Die Anbaugebiete Finnlands. Acta agr. fenn. 47: $1-147$.

(15) - - 1942. Kasvinviljelys. 278 s. Helsinki.

(16) - - 1944. Lisiä peltoherneen viljelysominaisuuksien tuntemiseen. Maa 29: 152-155.

(17) - 1955. Protein content of field pea seeds as a varietal character. Acta agr. fenn. 83 $125-132$.

(18) - - 1957. On quality breeding of the field pea and its results. Selostus: Peltoherneen laatujalostuksesta ja sen tuloksista maatalouskoelaitoksen kasvinjalostusosastolla Jokioisissa. Valt. maatal.koetoim. julk. 157: 1-23.

(19) Ронјакаllı, O. 1943. Uber die Abhängigkeit der Resistenz gegen die Trockenperiode und der Reifesicherheit von Entwicklungsrhythmus bei Hafer, Gerste und Sommerweizen. Selostus: Kauran, ohran ja kevätvehnän poudankestävyyden ja tuleentumisvarmuuden riippuvaisuudesta niiden kehityksen rytmistä. Maatal.tiet. aikak. 15: 105-125.

(20) - 1951. Uber den Einfluss der Umweltfaktoren auf die Dauer der Zeit von der Aussaat bis zum Ähren- (Rispen-) Schieben bei Sommergetreide. Soc. Scient. Fenn. Comment. Biol. XI. 6: 1-18.

(21) - - \& SAlonen, A. \& AntilA, S. 1961. Kevätviljan kehityksestä Helsingin yliopiston Viikin $\left(60^{\circ} 10^{\prime} \mathrm{N}\right)$ ja Muddusniemen $\left(69^{\circ} 5^{\prime} \mathrm{N}\right)$ koetiloilla suoritetuissa kenttäkokeissa. Referat: Uber die Entwicklung von Sommergetreide in Feldversuchen ausgeführt auf den Versuchsgütern der Universität Helsinki, Viik $\left(60^{\circ} 10^{\prime}\right)$ und Muddusniemi $\left(60^{\circ} 5^{\prime}\right)$. Maatal.tiet. aikak. 33: $65-80$.

(22) Ронјалнегмo, O. 1959. Lämpö- ja sadeolojen vaikutuksesta kevätviljoihin Jokioisissa 1930 - 54. Referat: Einfluss der Temperatur und der Niederschlagshöhe auf die Entwicklung der Sommergetreide in Jokioinen in den Jahren 1930-54. Maatal. ja koetoim. 13: 87-97.

(23) SALminen, M. 1930. Kasvuajan sademäărăn ja keskilämpötilan vaikutus lämpöasteiden summaan ja kasvuajan pituuteen Tammistossa 1925-29. Hankkijan Siemenjulkaisu 1930: 87-92.

(24) Sırı, S. S. 1926. Lämpöasteiden ja tehoisien lämpöasteiden summa sekä niiden merkitys kasvukauden pituuden määräämisessä. Maatalous 19: 169-171.

(25) Sinisalo, J. 1937. Die für das Reifen gewisser Sommerweizen- und Gerstensorten erforderlichen Wärmesummen und Anzahlen von Wachstumstagen. Selostus: Eräiden kevätvehnien ja ohrien tuleentumiseen tarvittavat kasvupäivien luvut ja lämpöasteiden summat. Maatal. tiet. aikak. 9: 194-212.

(26) Valtion Siementarkastuslaitos. Toimintakertomukset vuosilta 1938-1960. Helsinki. 


\title{
SÄTEKIJÖIDEN VAIKUTUS HERNEEN KEHITYKSEEN
}

\author{
K. MULtamäKI
}

Maatalouden Tutkimuskeskus, kasvinjalostuslaitos, Jokioinen

Tutkimus perustuu Torstai II-herneestä Jokioisissa v. 1929-60 eli 32 vuoden aikana kertyneeseen koetulosaineistoon. Tämän lajikkeen kasvuaika (W) jaettiin kahteen jaksoon, joista ensimmäinen (I) käsitti ajan kylvöpäivästä kukinnan alkamiseen ja toinen (II) loppuosan kasvuajasta tuleentumispäivään saakka. Kummallekin jaksolle ja koko kasvuajalle laskettiin vuotuiset ja keskimääräiset kestämisajat päivissä, keskilämpötilat, sadesummat ja tehoisien lämpöasteiden summat (taulukko 1). Tehoisia lämpöasteita laskettaessa vähennettiin päivän keskilämpötilasta $4^{\circ} \mathrm{C}$.

Torstai II-herneen kehityksen rytmiin vaikuttaneita säätekijöitä tarkasteltaessa (taulukko 1) ilmenee, että keskilämpötilat ovat muunnelleet verraten vähän, sademäärät sitä vastoin varsin paljon. Lämpösummat muuntelivat vähemmän kuin kasvupäivien lukumäärät. Toisen jakson lämpösummien ja kasvupäivien muuntelu oli voimakkaampaa kuin ensimmäisen jakson ja koko kasvuajan.

Koetulosaineistossa mahdollisesti esiintyviä vuorosuhteita selvitettäessä laskettiin 37 korrelaatiokerrointa (taulukko 2). Myöhäinen kylvö lyhensi selvästi ensimmäisen jakson pituutta. Kummankin jakson sekä koko kasvuajan keskilämpötila oli negatiivisessa vuorosuhteessa niiden kestämiasaikaan. Toisen jakson ja koko kasvuajan sadesumman ja tehoisien lämpöasteiden summan puolestaan todettiin olevan positiivisessa vuorosuhteessa niiden kestämisaikaan. Kasvuajan sadesumma osoittautui olevan positiivisessa vuorosuhteessa kasvuajan tehoisien lämpöasteiden summaan nähden.

Siemensadon määrään liittyviä korrelaatiokertoimia laskettiin kaikkiaan 14. Kylvöajan ja säätekijöiden muuntelun ei todettu tilastollisella varmuudella vaikuttavan siemensadon määrään. Toisaalta kuitenkin ilmeni ensimmäisen jakson sekä koko kasvuajan pituuksien ja siemensadon välillä positiiviset vuorosuhteet. Varsisadon määrän todettiin olevan positiivisessa vuorosuhteessa sekä kasvuajan pituuteen että sen sadesummaan ja tehoisien lämpöasteiden summaan. Varsi- ja siemensatojen välillä vallitsi positiivinen korrelaatio. Kasvuston korkeus ja laonkestävyys olivat keskenään negatiivisessa korrelaatiossa. Siemenen koon ja käyttöominaisuuksien kesken ei todettu merkitseviä vuorosuhteita. 